\title{
Balanced Semi-Latin Rectangles: Properties, Existence and Constructions for Block Size Two
}

\author{
N. P. Uto ${ }^{1}$ (D) R. A. Bailey ${ }^{1}$ (D)
}

Published online: 8 July 2020

(c) The Author(s) 2020

\begin{abstract}
There exists a set of designs which form a subclass of semi-Latin rectangles. These designs, besides being semi-Latin rectangles, exhibit an additional property of balance, where no two distinct pairs of symbols (treatments) differ in their concurrences, that is, each pair of distinct treatments concurs a constant number of times in the design. Such a design exists for a limited set of parameter combinations. We designate it a balanced semi-Latin rectangle and give some properties and necessary conditions for its existence. Furthermore, algorithms for constructing the design for experimental situations where there are two treatments in each row-column intersection (block) are also given.
\end{abstract}

Keywords Balanced incomplete block design (BIBD) $\cdot$ Balanced semi-Latin rectangle $(\mathrm{BSLR}) \cdot$ Optimal design · Quotient block design (QBD) · Regular graph design (RGD)

\section{Introduction}

Semi-Latin rectangles form an important class of row-column designs with interesting and attractive combinatorial properties. Row-column designs admit two systems of blocks: the rows and columns, hence control heterogeneity in the experimental units which could have some effect on the response, in two directions: see, for example, [1, 16, 17] and [24, Chapter 5] for discussions on row-column designs.

Most of the classical row-column designs, which include, among others, Latin squares, Youden squares and generalized Youden designs, have only one plot in each row-column intersection (cell); hence, just one treatment can be applied to each cell: see, for example, $[2,11]$ and [31, Chapter 1] for several discussions on Latin

\section{N. P. Uto}

npu@st-andrews.ac.uk

1 School of Mathematics and Statistics, Mathematical Institute, University of St Andrews, North Haugh, St Andrews, Fife KY16 9SS, Scotland 
squares; [29, 35] and [31, Chapter 6] for discussions on Youden squares; and [3, 25, 34] for generalized Youden designs.

An immediate generalization of the Latin square (LS) is the semi-Latin square (SLS), which is further generalized by the semi-Latin rectangle (SLR). In a SLS, the treatments are arranged in a square array, where each row-column intersection (block) contains more than one treatment. The Latin square and semi-Latin square have a property that each treatment is restricted to appearing only once in each row and also only once in each column; hence, for each of them, the number of rows is identical to the number of columns, and the number of treatments is simply the product of the block (cell) size and the number of rows (or columns). The semiLatin square has an advantage over the Latin square in the sense that it can accommodate more than one treatment in each row-column intersection. For discussions on semi-Latin squares, see, for example, [4-6, 8, 9, 28, 30, 33, 36].

If there exists a set of $k$ mutually orthogonal Latin squares of order $s$, where $2 \leq k \leq s-1$, then they may be superimposed to obtain a semi-Latin square with $s$ rows and columns and $k$ treatments per cell. Such a semi-Latin square is called a Trojan square by [15]. Although the case $k=2$ was excluded by [15], more recent literature, such as [18], includes it.

When $k=2$, the pair of mutually orthogonal Latin squares is often called a Graeco-Latin square: see [13, Chapter 6] and [31, Chapter 1]. However, this is not the same thing as the Trojan square. If the two constituent Latin squares have Latin and Greek letters, respectively, then they might be used for a design in which one treatment factor has $s$ levels given by the Latin letters and a second treatment factor has $s$ levels given by the Greek letters. On the other hand, the Trojan square simply has one treatment factor with $2 s$ levels. If $k=s-1$, the set of mutually orthogonal Latin squares is called a hyper-Graeco-Latin square in [10]; see also [31, Chapter 1]; again, this is not the same as the Trojan square.

The treatment concurrences in a Trojan square are 0 and 1 , and when such design exists it is known to be optimal among semi-Latin squares of its size, and indeed among all incomplete block designs for $s k$ treatments in $s^{2}$ blocks of size $k$ : see [14].

However, there could be certain experimental situations where the within-row replication number, as well as the within-column replication number, of each treatment is not limited to 1 . In such circumstances, the semi-Latin rectangle, which generalizes both the Latin square and the semi-Latin square, becomes an invaluable design. This was introduced in [7]. The balanced semi-Latin rectangle (BSLR) is a special case.

In a SLR, there are $v$ treatments, $h$ rows, $p$ columns and $k$ treatments in each row-column intersection. We denote this structure by $(h \times p) / k$. Each row contains each treatment a constant number, $n_{r}$, of times. Similarly, each column contains each treatment a constant number, $n_{c}$, of times. In the general case, $h$ and $p$ are not necessarily distinct; likewise $n_{r}$ and $n_{c}$.

The quotient block design (QBD) of a SLR is the block design obtained by ignoring its rows and columns. For a SLR, we impose the condition that the QBD is binary. BSLRs form a subclass of semi-Latin rectangles with the property that their quotient block designs form balanced incomplete block designs (BIBDs). BIBDs are binary, proper and equireplicate incomplete block designs with a unique number 
of concurrences between every pair of treatments: see, for example, [26], and [32, Chapter 4] for some descriptions and discussions on BIBDs. BSLRs are, indeed, semi-Latin rectangles with an additional property of balance.

However, balanced designs do not exist for all values of the parameters; hence, their existence is limited. When they exist, they are known to possess optimality properties; they are optimal within their class over a range of criteria. For instance, a BIBD is optimal over all incomplete block designs of the same size. For such a design, its canonical efficiency factors are identical, and all elementary contrasts of treatment effects are estimated with the same variance: see, for example, [23, 25].

Consequently, the existence of a BSLR is also restricted to certain sets of combinations of the parameters. But when such a design exists, it is, indeed, optimal over all semi-Latin rectangles in its class.

Semi-Latin rectangles have been found useful for conducting several types of experiment. Among these are agricultural experiments, such as plant disease experiments; food sensory experiments; and consumer testing experiments. For instance, a $(4 \times 8) / 2$ SLR, involving eight plants $(p=8)$ and pairs of half-leaves $(k=2)$ at four heights $(h=4)$, with eight treatments $(v=8)$, where each was a solution made from an extract of one of the offspring plants, was used for an experiment on tobacco plants, regarding mosaic virus, at Rothamsted Experimental Station: see [7].

In food sensory experiments, there are $p$ panellists, $h$ food-tasting sessions and $v$ different food items (treatments) available for tasting; and each panellist is made to taste $k$ items of food in each of $h$ sessions. Again, for consumer testing experiments, $v$ brands of a given consumer good (treatments) are available for the experiment; and these are to be tested by $p$ consumers in $h$ time periods, weeks, say. Each consumer tests $k$ brands of the product each week: see, for example, [7]. Moreover, [5] describes some other experimental situations where a semi-Latin square can be used. In similar experimental situations, a semi-Latin rectangle becomes a useful design.

Balanced semi-Latin rectangles, when they exist, would be the best in any of the experimental situations described above.

Constructions are given in [7] for efficient SLRs for $2 n$ treatments in $n$ rows and $2 n$ columns, where $2 \leq n \leq 10$, using some combinatorial approaches, viz starters and the cyclic method for constructing balanced tournament designs. Each row-column intersection (block) contains two treatments; and each treatment appears once in each column, but twice in each row. This is, simply, an $(n \times 2 n) / 2$ SLR corresponding to $h=n, p=2 n$ and $k=2$. Each design is group-divisible, and indeed, but with the exception of the case $n=2$, a regular graph semi-Latin rectangle (RGSLR). The designs are group-divisible in the sense that their treatments can be partitioned into groups (subsets), each being of a constant size, and each pair of treatments in the same group concurs a constant number of times. Similarly, each pair of treatments in different groups concurs a constant number of times also. Furthermore, the designs are regular graph semi-Latin rectangles since no two pairs of distinct treatments differ in their concurrences by more than unity, in absolute terms. In particular, the treatment concurrences in these designs are 1 and 2 . The search for regular graph semi-Latin rectangles in [7] is buttressed by the belief that, when a BIBD does not exist, there will be 
a regular graph design (RGD) which is an optimal design within its class, particularly when the number of blocks is large enough: see, for example, [12, 22, 23].

In this work, we introduce the design, balanced semi-Latin rectangle, which forms a subclass of semi-Latin rectangles, as noted earlier. Section 2 is concerned with some definitions, combinatorial properties of the design and some necessary conditions on the parameters for such a design to exist. Section 3 gives some methods of construction for the case where there are two treatments in each row-column intersection.

As in [7], for the constructions we consider only BSLRs for the case that $k=2$ because this seems likely to be very useful in practice. But unlike [7], we allow $h \geq p$ since given an $(h \times p) / k$ SLR where $h<p$, a corresponding $(p \times h) / k$ SLR with $p>h$ can be obtained by transposition. Also, we allow $h=p$; though this looks like a square, we call it a rectangle because the standard definition of semi-Latin square includes the condition that $n_{r}=n_{c}=1$.

\section{Definitions, Combinatorial Properties and Conditions for Existence}

\subsection{Definitions}

Definition 1 Let $v, h, p, k, n_{r}$ and $n_{c} \in \mathbb{Z}_{+}$. Suppose $h, p>1 ; v$ divides $k h$ and $v$ divides $k p$. Then, an $(h \times p) / k$ semi-Latin rectangle (SLR) is a row-column design in which $v$ treatments are arranged into $h$ rows and $p$ columns, where each row-column intersection (block) contains $k$ treatments (each treatment appearing at most once in each block), and each treatment appears a constant number, $n_{r}$, of times in each row, and also a constant number, $n_{c}$, of times in each column.

Remark The definition of semi-Latin rectangle given in [7] does not accommodate $h=p$, as $h<p$ is assumed; and by virtue of this, $n_{r}>n_{c}$.

Figure 1 shows an example for $v=8, h=4, p=8$ and $k=2$. This example was adapted from [7]. The design, though non-balanced, is a regular graph semi-Latin rectangle.

Definition 2 Let $\Omega$ denote a semi-Latin rectangle, and $\Delta(\Omega)$ its quotient block design. Denote by $\mathcal{V}=\{1,2, \ldots, v\}$ its treatment set; and $\lambda_{i i^{\prime}}, i \neq i^{\prime}$, the number of concurrences between treatments $i$ and $i^{\prime}$. Suppose $\Delta(\Omega)$ forms a balanced incomplete block design, in the sense that, in addition to having each treatment appearing in $h n_{r}=p n_{c}$ blocks (which is basic to semi-Latin rectangles), there exists a constant,

\begin{tabular}{|ll|ll|ll|ll|ll|ll|ll|ll|}
\hline 1 & 4 & 2 & 5 & 3 & 6 & 4 & 7 & 5 & 8 & 6 & 1 & 7 & 2 & 8 & 3 \\
\hline 2 & 8 & 3 & 1 & 4 & 2 & 5 & 3 & 6 & 4 & 7 & 5 & 8 & 6 & 1 & 7 \\
\hline 3 & 7 & 4 & 8 & 5 & 1 & 6 & 2 & 7 & 3 & 8 & 4 & 1 & 5 & 2 & 6 \\
\hline 5 & 6 & 6 & 7 & 7 & 8 & 8 & 1 & 1 & 2 & 2 & 3 & 3 & 4 & 4 & 5 \\
\hline
\end{tabular}

Fig. 1 A $(4 \times 8) / 2$ regular graph (non-balanced) semi-Latin rectangle for 8 treatments 
$\lambda$ such that $\lambda_{i i^{\prime}}=\lambda$ for all $i, i^{\prime} \in \mathcal{V}, i \neq i^{\prime}$. Then, $\Omega$ is said to be a balanced semi-Latin rectangle; otherwise, it is a non-balanced semi-Latin rectangle.

\subsection{Combinatorial Properties}

A balanced semi-Latin rectangle, just like other semi-Latin rectangles, exhibits the property of orthogonality with respect to the row and column strata. Its treatments are orthogonal to the row stratum, as well as the column stratum: see [7]. The treatments of this design are orthogonal to the row stratum, in the sense that each treatment appears the same number, $n_{r}$, of times in each row. Similarly, the treatments are orthogonal to the column stratum since each treatment appears the same number, $n_{c}$, of times in each column, where $n_{r}$ and $n_{c}$ are not necessarily equal. In particular, given an $(h \times p) / k$ SLR on $v$ treatments, $n_{r}=k p / v$, and $n_{c}=k h / v$. Thus, $v$ divides $k h$ and $v$ divides $k p$, and so

$$
k h \equiv 0(\bmod v)
$$

and

$$
k p \equiv 0(\bmod v) .
$$

Hence, $h n_{r}=p n_{c}$, which gives the number of times each treatment appears, overall, in the design.

Moreover, overall, there are $k h p$ plots in the design. Since each treatment is replicated $h n_{r}=p n_{c}$ times in the design, then

$$
v h n_{r}=v p n_{c}=k h p .
$$

We note that $n_{r}=n_{c}$ if and only if $p=h$. Similarly, $n_{r}>n_{c}$ if and only if $p>h$, and $n_{r}<n_{c}$ if and only if $p<h$.

Moreover, the design is $n_{r^{-}}$and $n_{c}$-resolvable with regards to the rows and columns, respectively. It is $n_{r}$-resolvable in the sense that, in each row which constitutes a superblock, each treatment appears exactly $n_{r}$ times. Similarly, the design is $n_{c^{-}}$ resolvable since each treatment appears $n_{c}$ times in each column, which constitutes another superblock.

Furthermore, each pair of distinct treatments concurs a constant number of times in the design. This is what distinguishes a BSLR from general SLRs. Denoting the constant number of concurrences between each pair of distinct treatments in the design by $\lambda$, each treatment appears with each of $v-1$ distinct complementary treatments $\lambda$ times. Also, each treatment appears in $h n_{r}=p n_{c}$ blocks, overall, and in each of these blocks it concurs with $k-1$ distinct treatments. Hence, the sum of concurrences with that treatment is $\lambda(v-1)$, so

$$
\lambda(v-1)=h n_{r}(k-1)=p n_{c}(k-1) .
$$

Also, $v \in(k, h p]$, by virtue of both Fisher's inequality and the blocks of the design being incomplete. 


\subsection{Existence of the Design}

Equations (1) and (2) give necessary conditions for a BSLR to exist.

Let $\mathcal{V}=\{1,2, \ldots, v\}$ be the set of treatments of a $(v, k, \lambda)$-BIBD, where $k \in[2, v)$ is the size of each block and $\lambda$ is the concurrence number between every possible pair of treatments. Then,

$$
\lambda(v-1) \equiv 0(\bmod k-1)
$$

and

$$
\lambda v(v-1) \equiv 0(\bmod k(k-1)) .
$$

Equation (2) implies Eq. (3). Equations (1) and (2) together imply Eq. (4). Hence, Eqs. (3) and (4) give necessary conditions for the existence of a BIBD: see, for example, [21,27].

The conditions in Eqs. (3) and (4) are not, in general, sufficient, in the sense that there exist certain situations where these conditions, though satisfied, may not guarantee the existence of a design. For instance, a $(15,5,2)$-BIBD does not exist, though its parameters satisfy Eqs. (3) and (4): see, for example, [24, Chapter 1], and [20]. Again, the existence of a $(22,8,4)$-BIBD, whose parameters also satisfy Eqs. (3) and (4), is uncertain: see, for example, [37, Chapter 1].

However, for $k \in[2,5]$ and all possible values of $\lambda$, except for the aforementioned $(15,5,2)$-BIBD, the conditions in Eqs. (3) and (4) are sufficient for the existence of a BIBD: see, for example, [19-21]. Also, for $k=6$ and $\lambda>1$, except for the (21, 6, 2)-BIBD, Eqs. (3) and (4) are also sufficient for a BIBD to exist: see, for example, [21]. For more results on the sufficiency (or otherwise) of Eqs. (3) and (4), see, for example, [21, 38].

\section{Construction of the Designs}

We base our constructions on an algebraic approach via addition on the set of integers modulo $v$ (or modulo $v-1$ ), as the case may be. Note that we regard the set of integers modulo $v$ as $\{1,2, \ldots, v\}$.

\subsection{Case 1: When $v$ is 0 dd}

Label the treatments by the integers modulo $v$. Put $\delta=(v-1) / 2$. For $u=1, \ldots, \delta$ and $j=1, \ldots, v$, put $S_{u j}=\{j, j+u\}$.

Notice that $S_{u 1}=\{1,1+u\}$. Similarly, $S_{1 j}=\{j, j+1\}, S_{2 j}=\{j, j+2\}$, and so on, each component reduced modulo $v$.

We now give an algorithm for constructing the design. 


\subsubsection{An Algorithm for Constructing the Design When $v$ is Odd}

Step 1: For $u=1, \ldots, \delta$ and $j=1, \ldots, v$, put $S_{u j}=\{j, j+u\}$, where each component is reduced modulo $v$.

Step 2: For $u=1, \ldots, \delta$, make a Latin square, $\Delta_{u}$, of order $v$ using the sets $S_{u 1}, S_{u 2}, \ldots, S_{u v}$, as symbols.

Step 3: Juxtapose the Latin squares $\Delta_{1}, \Delta_{2}, \ldots, \Delta_{\delta}$ made in Step 2, one beside another.

This algorithm produces a $(v \times v \delta) / 2$ BSLR. The constructed design is identical to:

\begin{tabular}{|l||l||l||l|}
\hline$\Delta_{1}$ & $\Delta_{2}$ & $\cdots$ & $\Delta_{\delta}$ \\
\hline
\end{tabular}

where $\Delta_{u}, u=1,2, \ldots, \delta$, could be the Latin square

$\Delta_{u}=$\begin{tabular}{|c|c|c|c|}
\hline$S_{u 1}$ & $S_{u 2}$ & $\cdots$ & $S_{u v}$ \\
\hline$S_{u v}$ & $S_{u 1}$ & $\cdots$ & $S_{u, v-1}$ \\
\hline$\vdots$ & $\vdots$ & $\ddots$ & $\vdots$ \\
\hline$S_{u 2}$ & $S_{u 3}$ & $\cdots$ & $S_{u 1}$ \\
\hline
\end{tabular}

Example 1 We illustrate the construction in Fig. 2, obtained via the algorithm. In this example, $v=h=5, \delta=2$, and $p=10$.

\section{Comments}

(1) The double vertical lines show the construction and should be ignored in randomization.

(2) The Latin square, $\Delta_{u}$, does not need to be cyclic.

(3) Also, $\Delta_{u}$ does not need to be isomorphic to $\Delta_{u^{\prime}}$, where $u \neq u^{\prime}$.

(4) If $\delta=1$, then $v=3$, and we have $a(3 \times 3) / 2$ BSLR as the only possible design.

\begin{tabular}{|ll|ll|ll|ll|ll||ll|ll|ll|ll|ll|}
\hline 1 & 2 & 2 & 3 & 3 & 4 & 4 & 5 & 5 & 1 & 1 & 3 & 2 & 4 & 3 & 5 & 4 & 1 & 5 & 2 \\
\hline 5 & 1 & 1 & 2 & 2 & 3 & 3 & 4 & 4 & 5 & 5 & 2 & 1 & 3 & 2 & 4 & 3 & 5 & 4 & 1 \\
\hline 4 & 5 & 5 & 1 & 1 & 2 & 2 & 3 & 3 & 4 & 4 & 1 & 5 & 2 & 1 & 3 & 2 & 4 & 3 & 5 \\
\hline 3 & 4 & 4 & 5 & 5 & 1 & 1 & 2 & 2 & 3 & 3 & 5 & 4 & 1 & 5 & 2 & 1 & 3 & 2 & 4 \\
\hline 2 & 3 & 3 & 4 & 4 & 5 & 5 & 1 & 1 & 2 & 2 & 4 & 3 & 5 & 4 & 1 & 5 & 2 & 1 & 3 \\
\hline
\end{tabular}

Fig. 2 A $(5 \times 10) / 2$ balanced semi-Latin rectangle for 5 treatments 
If $\delta>1$, we could obtain another possibility by transposition. If $\delta$ is prime, then this transposed design is the only other possibility; otherwise, if there exists $a, b \in \mathbb{Z}$, where $1<a, b<\delta$ such that $a b=\delta$, a slight modification of Step 3 gives another possibility for all such $(a, b)$, which is an $\left(h^{\prime} \times p^{\prime}\right) / 2 B S L R$, where $h^{\prime}=a v$, and $p^{\prime}=b v$. In particular, if $a=b$, then $\delta=a^{2}$, and consequently, $h^{\prime}=p^{\prime}=a v$.

Example 2 Let $v=9$. Then, $\delta=4$, and we can put $a=b=2$ and obtain the $(18 \times 18) / 2$ BSLR in Fig. 3 .

This is an alternative to a $(9 \times 36) / 2 \mathrm{BSLR}$ and a $(36 \times 9) / 2 \mathrm{BSLR}$ that could also be obtained for the same values of $v$ and $\delta$.

Again, the double vertical and horizontal lines show construction but are not part of the design.

\subsection{Case 2: When $v$ is Even}

Label one of the treatments $\infty$, and the others by the integers modulo $v-1$. Put $t=v / 2$. For $m=1, \ldots, t$ and $l=1, \ldots, v-1$, put

$$
A_{l m}= \begin{cases}\{l, \infty\}, & \text { if } m=1, \\ \{l+m-1, l-m+1\}, & \text { if } m \in(1, t],\end{cases}
$$

with reduction modulo $v-1$ for each component. In particular, $A_{l 1}=\{l, \infty\}$.

We give, in the next section, an algorithm for constructing the design.

\begin{tabular}{|c|c|c|c|c|c|c|c|c|c|c|c|c|c|c|c|c|c|c|c|c|c|c|c|c|c|c|c|c|c|}
\hline 2 & 2 & 3 & & 4 & & & & 7 & 7 & 8 & 8 & & & & 3 & \begin{tabular}{|l|}
2 \\
\end{tabular} & 4 & 3 & & 4 & & 7 & & 8 & & 9 & 8 & 1 & \\
\hline 1 & 2 & $\overline{2}$ & & 4 & & 45 & & 6 & 6 & 7 & 7 & 8 & 9 & & 2 & 1 & 3 & 2 & 4 & 35 & & 6 & 5 & 7 & & 8 & 7 & 9 & 8 \\
\hline 9 & 1 & 1 & 2 & 2 & & 34 & & 5 & 5 & 6 & 6 & 7 & 8 & & 1 & 9 & 2 & 1 & 3 & 2 & & 5 & & 6 & & 7 & 6 & 8 & 7 \\
\hline 8 & 9 & 9 & 1 & 2 & & $\begin{array}{ll}2 & 3\end{array}$ & 3 & 4 & 4 & 5 & 6 & 5 & 7 & & 9 & 8 & 1 & 9 & 2 & 1 & & 4 & 3 & 5 & & 6 & 5 & 7 & 6 \\
\hline 7 & 8 & 8 & & 9 & & 12 & 2 & $\begin{array}{ll}2 & 3\end{array}$ & 3 & 4 & 5 & & 6 & & 8 & 7 & 9 & 8 & 1 & $\begin{array}{ll}9 & 2\end{array}$ & & 13 & 2 & 4 & & 5 & 4 & 6 & 5 \\
\hline 6 & 7 & 7 & & 8 & & $\begin{array}{ll}9 & \end{array}$ & 1 & 2 & 2 & 3 & 3 & 4 & 5 & & 7 & 6 & 8 & 7 & 9 & $\begin{array}{ll}81 \\
\end{array}$ & & $\begin{array}{ll}92 \\
\end{array}$ & & 3 & & 4 & 3 & 5 & 4 \\
\hline 5 & 6 & 6 & 7 & 8 & & 89 & 9 & $\begin{array}{ll}9 & 1\end{array}$ & 1 & 2 & 3 & 3 & 4 & & 6 & 5 & 7 & 6 & 8 & $\begin{array}{ll}7 & 9\end{array}$ & & $\begin{array}{ll}81 \\
\end{array}$ & $\overline{9}$ & 2 & & 3 & 2 & 4 & \\
\hline 4 & 4 & 5 & 6 & 6 & & 78 & 8 & $\begin{array}{ll}89 \\
\end{array}$ & 9 & 1 & 1 & 2 & 23 & & 35 & 4 & 6 & 5 & 7 & 68 & & $\begin{array}{ll}7 & 9\end{array}$ & & 1 & g & 2 & 1 & 3 & 2 \\
\hline 3 & 3 & 4 & & 5 & & $\begin{array}{ll}6 & 7 \\
\end{array}$ & 7 & 8 & 8 & 9 & 9 & 1 & 2 & & 24 & 3 & 5 & 4 & 6 & $\begin{array}{ll}5 & 7 \\
\end{array}$ & & $\begin{array}{ll}68 \\
\end{array}$ & & 9 & & 1 & 9 & 2 & \\
\hline 4 & 2 & 3 & 6 & 4 & & 5 & 6 & 9 & 7 & 1 & & 2 & 3 & & 5 & 2 & & 3 & 7 & $\begin{array}{ll}4 & 8 \\
\end{array}$ & & & & 1 & & & 8 & 3 & \\
\hline 3 & 1 & 2 & 5 & 3 & & 47 & 5 & 8 & 6 & 9 & 7 & 1 & 2 & & 4 & 1 & 5 & 2 & & $\begin{array}{ll}3 & 7\end{array}$ & & 48 & 5 & 69 & & 1 & 7 & 2 & 8 \\
\hline & 3 & 1 & 4 & 2 & & 36 & 4 & 47 & 5 & 8 & 6 & 9 & 1 & & $\begin{array}{ll}3 & 3\end{array}$ & 9 & 4 & 1 & 5 & $\begin{array}{ll}2 & 6\end{array}$ & & $\begin{array}{ll}37 \\
\end{array}$ & & 18 & & 9 & 6 & 1 & 7 \\
\hline 1 & 8 & 9 & & 1 & & $\begin{array}{ll}25 \\
\end{array}$ & & 6 & 4 & & 5 & 8 & 9 & & 72 & 8 & 3 & 9 & 4 & $\begin{array}{ll}1 & 5 \\
\end{array}$ & & 26 & 3 & 7 & & 8 & 5 & 9 & 6 \\
\hline 9 & 7 & 8 & 2 & 9 & & 1 & 2 & 5 & 3 & 6 & 4 & 7 & 8 & & 1 & 7 & 2 & 8 & 3 & \begin{tabular}{|ll}
9 & 4 \\
\end{tabular} & & 15 & 2 & 6 & & 7 & 4 & 8 & 5 \\
\hline 8 & 6 & 7 & 1 & 8 & & 3 & 1 & 4 & 2 & 5 & 3 & 6 & 7 & & 9 & 6 & 1 & 7 & & $\begin{array}{|ll|}8 & 3 \\
\end{array}$ & & \begin{tabular}{|ll}
9 & 4 \\
\end{tabular} & & 5 & & 6 & 3 & 7 & 4 \\
\hline 7 & 5 & 6 & & 7 & & $\begin{array}{ll}82 \\
\end{array}$ & 9 & 3 & 1 & 4 & 2 & 5 & 6 & & 8 & 5 & 9 & 6 & 1 & 7 & & 83 & 9 & 4 & 1 & 5 & 2 & 6 & 3 \\
\hline & 4 & 5 & 8 & 6 & & 1 & 8 & 2 & 9 & 3 & 1 & 4 & 5 & & 7 & 4 & 8 & 5 & & \begin{tabular}{|ll}
6 & 1 \\
\end{tabular} & & $\begin{array}{ll}72 \\
\end{array}$ & 8 & 33 & & 4 & 1 & 5 & $\begin{array}{ll}2 & 6 \\
\end{array}$ \\
\hline 25 & 36 & 4 & & $\begin{array}{ll}5 & 8\end{array}$ & & & & & 8 & & $\begin{array}{ll}9 & 3\end{array}$ & & & & & 3 & 7 & & & & & & & & & & 9 & & \\
\hline
\end{tabular}

Fig. 3 An $(18 \times 18) / 2$ balanced semi-Latin rectangle for 9 treatments 


\subsubsection{An Algorithm for Constructing the Design When $v$ is Even}

Step 1: For $m=1, \ldots, t$ and $l=1, \ldots, v-1$, put

$$
A_{l m}= \begin{cases}\{l, \infty\}, & \text { if } m=1, \\ \{l+m-1, l-m+1\}, & \text { if } m \in(1, t]\end{cases}
$$

each component being reduced modulo $v-1$.

Step 2: Make a Latin square $\Xi_{l}$, of order $t$, for $l=1,2, \ldots, v-1$, using the sets $A_{l 1}$, $A_{l 2}, \ldots, A_{l t}$, as symbols.

Step 3: Juxtapose the Latin squares $\Xi_{1}, \Xi_{2}, \ldots, \Xi_{v-1}$ made in Step 2, one beside another.

This algorithm produces an $(h \times p) / 2$ BSLR, where $h=t$, and $p=t(v-1)$. The constructed design is simply:

\begin{tabular}{|l||l||l||l|}
\hline$\Xi_{1}$ & $\Xi_{2}$ & $\cdots$ & $\Xi_{v-1}$ \\
\hline
\end{tabular}

where each Latin square, $\Xi_{1}, \Xi_{2}, \ldots, \Xi_{v-1}$, is of order $t$ and could take any formcyclic or otherwise.

For instance, suppose they are cyclic, they could take the form:

$\Xi_{l}=$\begin{tabular}{|c|c|c|c|}
\hline$A_{l 1}$ & $A_{l 2}$ & $\cdots$ & $A_{l t}$ \\
\hline$A_{l t}$ & $A_{l 1}$ & $\cdots$ & $A_{l, t-1}$ \\
\hline$\vdots$ & $\vdots$ & $\ddots$ & $\vdots$ \\
\hline$A_{l 2}$ & $A_{l 3}$ & $\cdots$ & $A_{l 1}$ \\
\hline
\end{tabular}

Example 3 Figure 4 shows an example with $v=6, h=3$, and $p=15$.

Comment If v-1 is not prime, in a similar manner as in Case 1, there are other possibilities for the design. Suppose that there are $w, y \in \mathbb{Z}$, where $1<w, y<v-1$ and $w y=v-1$. Then, the design could have $h=w t$ and $p=y t$. If $w=y$, this gives $h=p=w t$.

\begin{tabular}{|rr|rr|rr||rr|rr|rr||rr|rr|rr||rr|rr|rr||rr|rr|rr|}
\hline 1 & $\infty$ & 2 & 5 & 3 & 4 & 2 & $\infty$ & 3 & 1 & 4 & 5 & 3 & $\infty$ & 4 & 2 & 5 & 1 & 4 & $\infty$ & 5 & 3 & 1 & 2 & 5 & $\infty$ & 1 & 4 & 2 & 3 \\
\hline 3 & 4 & 1 & $\infty$ & 2 & 5 & 4 & 5 & 2 & $\infty$ & 3 & 1 & 5 & 1 & 3 & $\infty$ & 4 & 2 & 1 & 2 & 4 & $\infty$ & 5 & 3 & 2 & 3 & 5 & $\infty$ & 1 & 4 \\
\hline 2 & 5 & 3 & 4 & 1 & $\infty$ & 3 & 1 & 4 & 5 & 2 & $\infty$ & 4 & 2 & 5 & 1 & 3 & $\infty$ & 5 & 3 & 1 & 2 & 4 & $\infty$ & 1 & 4 & 2 & 3 & 5 & $\infty$ \\
\hline
\end{tabular}

Fig. 4 A $(3 \times 15) / 2$ balanced semi-Latin rectangle for 6 treatments 


\begin{tabular}{|rr|rr|rr|rr|rr||rr|rr|rr|rr|rr||rr|rr|rr|rr|rr|}
\hline 1 & $\infty$ & 2 & 9 & 3 & 8 & 4 & 7 & 5 & 6 & 2 & $\infty$ & 3 & 1 & 4 & 9 & 5 & 8 & 6 & 7 & 3 & $\infty$ & 4 & 2 & 5 & 1 & 6 & 9 & 7 & 8 \\
\hline 5 & 6 & 1 & $\infty$ & 2 & 9 & 3 & 8 & 4 & 7 & 6 & 7 & 2 & $\infty$ & 3 & 1 & 4 & 9 & 5 & 8 & 7 & 8 & 3 & $\infty$ & 4 & 2 & 5 & 1 & 6 & 9 \\
\hline 4 & 7 & 5 & 6 & 1 & $\infty$ & 2 & 9 & 3 & 8 & 5 & 8 & 6 & 7 & 2 & $\infty$ & 3 & 1 & 4 & 9 & 6 & 9 & 7 & 8 & 3 & $\infty$ & 4 & 2 & 5 & 1 \\
\hline 3 & 8 & 4 & 7 & 5 & 6 & 1 & $\infty$ & 2 & 9 & 4 & 9 & 5 & 8 & 6 & 7 & 2 & $\infty$ & 3 & 1 & 5 & 1 & 6 & 9 & 7 & 8 & 3 & $\infty$ & 4 & 2 \\
\hline 2 & 9 & 3 & 8 & 4 & 7 & 5 & 6 & 1 & $\infty$ & 3 & 1 & 4 & 9 & 5 & 8 & 6 & 7 & 2 & $\infty$ & 4 & 2 & 5 & 1 & 6 & 9 & 7 & 8 & 3 & $\infty$ \\
\hline \hline 4 & $\infty$ & 5 & 3 & 6 & 2 & 7 & 1 & 8 & 9 & 5 & $\infty$ & 6 & 4 & 7 & 3 & 8 & 2 & 9 & 1 & 6 & $\infty$ & 7 & 5 & 8 & 4 & 9 & 3 & 1 & 2 \\
\hline 8 & 9 & 4 & $\infty$ & 5 & 3 & 6 & 2 & 7 & 1 & 9 & 1 & 5 & $\infty$ & 6 & 4 & 7 & 3 & 8 & 2 & 1 & 2 & 6 & $\infty$ & 7 & 5 & 8 & 4 & 9 & 3 \\
\hline 7 & 1 & 8 & 9 & 4 & $\infty$ & 5 & 3 & 6 & 2 & 8 & 2 & 9 & 1 & 5 & $\infty$ & 6 & 4 & 7 & 3 & 9 & 3 & 1 & 2 & 6 & $\infty$ & 7 & 5 & 8 & 4 \\
\hline 6 & 2 & 7 & 1 & 8 & 9 & 4 & $\infty$ & 5 & 3 & 7 & 3 & 8 & 2 & 9 & 1 & 5 & $\infty$ & 6 & 4 & 8 & 4 & 9 & 3 & 1 & 2 & 6 & $\infty$ & 7 & 5 \\
\hline 5 & 3 & 6 & 2 & 7 & 1 & 8 & 9 & 4 & $\infty$ & 6 & 4 & 7 & 3 & 8 & 2 & 9 & 1 & 5 & $\infty$ & 7 & 5 & 8 & 4 & 9 & 3 & 1 & 2 & 6 & $\infty$ \\
\hline \hline 7 & $\infty$ & 8 & 6 & 9 & 5 & 1 & 4 & 2 & 3 & 8 & $\infty$ & 9 & 7 & 1 & 6 & 2 & 5 & 3 & 4 & 9 & $\infty$ & 1 & 8 & 2 & 7 & 3 & 6 & 4 & 5 \\
\hline 2 & 3 & 7 & $\infty$ & 8 & 6 & 9 & 5 & 1 & 4 & 3 & 4 & 8 & $\infty$ & 9 & 7 & 1 & 6 & 2 & 5 & 4 & 5 & 9 & $\infty$ & 1 & 8 & 2 & 7 & 3 & 6 \\
\hline 1 & 4 & 2 & 3 & 7 & $\infty$ & 8 & 6 & 9 & 5 & 2 & 5 & 3 & 4 & 8 & $\infty$ & 9 & 7 & 1 & 6 & 3 & 6 & 4 & 5 & 9 & $\infty$ & 1 & 8 & 2 & 7 \\
\hline 9 & 5 & 1 & 4 & 2 & 3 & 7 & $\infty$ & 8 & 6 & 1 & 6 & 2 & 5 & 3 & 4 & 8 & $\infty$ & 9 & 7 & 2 & 7 & 3 & 6 & 4 & 5 & 9 & $\infty$ & 1 & 8 \\
\hline 8 & 6 & 9 & 5 & 1 & 4 & 2 & 3 & 7 & $\infty$ & 9 & 7 & 1 & 6 & 2 & 5 & 3 & 4 & 8 & $\infty$ & 1 & 8 & 2 & 7 & 3 & 6 & 4 & 5 & 9 & $\infty$ \\
\hline
\end{tabular}

Fig. $5 \mathrm{~A}(15 \times 15) / 2$ balanced semi-Latin rectangle for 10 treatments

Example 4 For instance, if $v-1=9$, and consequently, $v=10$, apart from a $(5 \times 45) / 2$ BSLR and a $(45 \times 5) / 2$ BSLR, a $(15 \times 15) / 2$ BSLR can also be obtained: see Fig. 5.

\section{Compliance with Ethical Standards}

Conflict of interest On behalf of all authors, the corresponding author states that there is no conflict of interest.

Open Access This article is licensed under a Creative Commons Attribution 4.0 International License, which permits use, sharing, adaptation, distribution and reproduction in any medium or format, as long as you give appropriate credit to the original author(s) and the source, provide a link to the Creative Commons licence, and indicate if changes were made. The images or other third party material in this article are included in the article's Creative Commons licence, unless indicated otherwise in a credit line to the material. If material is not included in the article's Creative Commons licence and your intended use is not permitted by statutory regulation or exceeds the permitted use, you will need to obtain permission directly from the copyright holder. To view a copy of this licence, visit http://creativecommons.org/licen ses/by/4.0/.

\section{References}

1. Agrawal H (1966) Some methods of construction of designs for two-way elimination of heterogeneity, 1. J Am Stat Assoc 61(316):1153-1171

2. Ai M, Li K, Liu S, Lin DKJ (2013) Balanced incomplete Latin square designs. J Stat Plan Inference 143:1575-1582

3. Ash A (1981) Generalized Youden designs: construction and tables. J Stat Plan Inference 5(1):1-25

4. Bailey RA (1988) Semi-Latin squares. J Stat Plan Inference 18:299-312

5. Bailey RA (1992) Efficient semi-Latin squares. Stat Sin 2:413-437

6. Bailey RA, Chigbu PE (1997) Enumeration of semi-Latin squares. Discrete Math 167/168:73-84

7. Bailey RA, Monod H (2001) Efficient semi-Latin rectangles: designs for plant disease experiments. Scand J Stat 28(2):257-270 
8. Bailey RA, Royle G (1997) Optimal semi-Latin squares with side six and block size two. Proc R Soc Ser A Math Phys Eng Sci 453:1903-1914

9. Bedford D, Whitaker RM (2001) A new construction for efficient semi-Latin squares. J Stat Plan Inference 98:287-292

10. Bose RC (1938) On the application of the properties of Galois fields to the problem of construction of hyper-Graeco-Latin squares. Sankhyā Indian J Stat 3(4):323-338

11. Bose RC, Nair KR (1941) On complete sets of Latin squares. Sankhyā Indian J Stat 5(4):361-382

12. Cakiroglu SA (2018) Optimal regular graph designs. Stat Comput 28:103-112

13. Cameron PJ (1994) Combinatorics: topics, techniques, algorithms. Cambridge University Press, Cambridge

14. Cheng C-S, Bailey RA (1991) Optimality of some two-associate-class partially balanced incomplete-block designs. Ann Stat 19(3):1667-1671

15. Darby LA, Gilbert N (1958) The Trojan square. Euphytica 7:183-188

16. Dash S, Parsad R, Gupta VK (2014) Efficient row-column designs with two rows. J Indian Soc Agric Stat 68(3):377-390

17. Datta A, Jaggi S, Varghese E, Varghese C (2017) Generalized confounded row-column designs. Commun Stat Theory Methods 46(12):6213-6221

18. Edmondson RN (1998) Trojan square and incomplete Trojan square designs for crop research. J Agric Sci 131:135-142

19. Hanani H (1961) The existence and construction of balanced incomplete block designs. Ann Math Stat 32(2):361-386

20. Hanani H (1972) On balanced incomplete block designs with blocks having five elements. J Comb Theory Ser A 12:184-201

21. Hanani H (1975) Balanced incomplete block designs and related designs. Discrete Math 11:255-369

22. John JA, Mitchell TJ (1977) Optimal incomplete block designs. J R Stat Soc Ser B (Methodol) 39(1):39-43

23. John JA, Williams ER (1982) Conjectures for optimal block designs. J R Stat Soc Ser B (Methodol) 44(2):221-225

24. John JA, Williams ER (1995) Cyclic and computer generated designs. Chapman and Hall, London

25. Kiefer J (1975) Balanced block designs and generalized Youden designs, I. Construction (patchwork). Ann Stat 3(1):109-118

26. Morgan JP (2007) Optimal incomplete block designs. J Am Stat Assoc 102(478):655-663

27. Nash-Williams CStJA (1972) Simple constructions for balanced incomplete block designs with block size three. J Comb Theory Ser A 13:1-6

28. Parsad R (2006) A note on semi-Latin squares. J Indian Soc Agric Stat 60(2):131-133

29. Preece DA (1996) Youden squares. In: Colbourn CJ, Dinitz JH (eds) The CRC handbook of combinatorial designs. CRC Press, Boca Raton, pp 511-514

30. Preece DA, Freeman GH (1983) Semi-Latin squares and related designs. J R Stat Soc Ser B (Methodol) 45(2):267-277

31. Raghavarao D (1971) Constructions and combinatorial problems in design of experiments. Wiley, New York

32. Raghavarao D, Padgett LV (2005) Block designs: analysis, combinatorics, and applications. World Scientific, Hackensack

33. Rojas B, White RF (1957) The modified Latin square. J R Stat Soc Ser B (Methodol) 19(2):305-317

34. Ruiz F, Seiden E (1974) On construction of some families of generalized Youden designs. Ann Stat 2(3):503-519

35. Shrikhande SS (1951) Designs for two-way elimination of heterogeneity. Ann Math Stat 22(2):235-247

36. Soicher LH (2013) Optimal and efficient semi-Latin squares. J Stat Plan Inference 143:573-582

37. Stinson DR (2004) Combinatorial designs: constructions and analysis. Springer, New York

38. Zhu L (1993) Some recent developments on BIBDs and related designs. Discrete Math 123:189-214

Publisher's Note Springer Nature remains neutral with regard to jurisdictional claims in published maps and institutional affiliations. 